\title{
Predicting significance of COX-2 expression of peripheral blood monocyte in patients with coronary artery disease
}

\author{
Qinghua Huang ${ }^{1}$, Xianming $\mathrm{Fei}^{2}$, Shengbing $\mathrm{Li}^{3}$, Chan $\mathrm{Xu}^{4}$, Chunping $\mathrm{Tu}^{5}$, Lei Jiang ${ }^{2}$, Mingyi $\mathrm{Wo}^{2}$ \\ ${ }^{1}$ Department of Endocrinology, ${ }^{2}$ Center for Laboratory Medicine, Zhejiang Provincial People's Hospital and People's Hospital of Hangzhou Medical \\ College, Hangzhou 310014, China; ${ }^{3}$ Department of Laboratory Medicine, The Second Jiaxing Hospital, Jiaxing 314000, China; ${ }^{4}$ Department of \\ Laboratory Medicine, Zhejiang Provincial Zhongshan Hospital, Hangzhou 330106, China; ${ }^{5}$ Department of Laboratory Medicine, Nanxun People's \\ Hospital of Huzhou, Huzhou 313009, China \\ Contributions: (I) Conception and design: X Fei, Q Huang; (II) Administrative support: X Fei; (III) Provision of study materials or patients: Q Huang, \\ M Wo; (IV) Collection and assembly of data: S Li, C Xu; (V) Data analysis and interpretation: All authors; (VI) Manuscript writing: All authors; (VII) \\ Final approval of manuscript: All authors. \\ Correspondence to: Xianming Fei. Center for Laboratory Medicine, Zhejiang Provincial People's Hospital and People's Hospital of Hangzhou Medical \\ College, 158 Shangtang Road, Xiacheng District, Hangzhou 310014, China. Email: fortunity@163.com.
}

Background: Cyclooxygenase-2 (COX-2) plays an important role in the monocyte-platelet aggregate (MPA)-medicated inflammatory response and possible coronary artery disease (CAD). This study aimed to assess the predicting significance of COX-2 expression in peripheral blood monocyte for CAD.

Methods: A total of 66 patients with CAD including stable angina (SA) and unstable angina (UA) were enrolled. The inflammatory indexes including white blood cell (WBC) count, high-sensitive $\mathrm{C}$ reactive protein (hs-CRP), serum monocyte chemoattractant protein-1 (MCP-1) and MPA levels were measured. The western-blotting assay and reverse transcription-polymerase chain reaction (RT-PCR) analysis were used to detect the COX-2 expression in peripheral blood monocytes. Furthermore, the correlation between COX-2 expression and MPA levels, and the association of COX-2 expression with CAD risk were assessed.

Results: The UA patients demonstrated higher levels of inflammatory indexes than the SA patients $(\mathrm{P}<0.001)$. Simultaneously, higher MPA levels and enhanced COX-2 expression were observed in the UA patients $(\mathrm{P}<0.01)$. The patients with enhanced COX-2 expression exhibited higher MPA than those without $(\mathrm{P}<0.01)$, and patients with increased MPA also demonstrated enhanced COX-2 expression $(\mathrm{P}<0.001)$. Moreover, the levels of COX-2 protein expression was positively related to the MPA formation rates $\left(\mathrm{R}^{2}=0.4933, \mathrm{P}<0.01\right)$, and enhanced COX-2 expression was independently associated with CAD risk [odds ratio (OR): 6.322, 95\% confidence interval (CI): 4.544-8.978 ].

Conclusions: The COX-2 expression of peripheral blood monocytes can be used as an independent predictor for CAD.

Keywords: Coronary artery disease (CAD); monocyte; cyclooxygenase-2 (COX-2); predictor

Submitted Apr 17, 2019. Accepted for publication Aug 12, 2019.

doi: $10.21037 /$ atm.2019.08.75

View this article at: http://dx.doi.org/10.21037/atm.2019.08.75

\section{Introduction}

Atherosclerosis is the major pathologic base of coronary artery disease (CAD). Patients with atherosclerosis will appear no symptoms at the beginning of atherosclerosis development, however, it can gradually progress into
CAD, which is regarded as the leading cause of deaths worldwide (1). Atherosclerotic process is highly associated with the inflammatory response in the endothelial cells of vessel wall which results in the generation of atheromatous plaques in the arterial tunica intima (2). Haynes et al. (3) reported that formation of monocyte-platelet aggregates 
(MPAs) is the major risk factor of cardiovascular events which may induce monocyte activation and the release of pro-inflammatory cytokines/molecules, and subsequently facilitates the occurrence of atherosclerosis. Therefore, the formation of MPA is an established link between inflammation and thrombosis in acute coronary syndromes and related disorders (4).

The binding of P-selectin to $\mathrm{P}$-selectin glycoprotein ligand type 1 (PSGL-1), the major way of MPA formation, can induce monocyte activation and the expression of cytokines, chemokines, and adhesion molecules, which subsequently results in the promotion of atherosclerotic lesion formation $(5,6)$. Under pro-inflammatory conditions, the enhancement of MPA subsequently expands the pool of circulating monocytes in a cyclooxygenase- 2 (COX-2) dependent manner (7). COX-2 can regulate prostaglandin E2 (PGE2) production by tumor necrosis factor- $\alpha$ (TNF- $\alpha$ ), and further promote the development and progression of chronic inflammation response (8). Furthermore, our previous study has revealed that the COX-2 expression induced by MPA formation and the release of monocyte chemoattractant protein-1 (MCP-1) in monocyte were downregulated by COX-2 inhibitor, and upregulated by TNF- $\alpha$ (9). Therefore, enhanced COX-2 expression in monocyte may play an important role in the MPA-medicated inflammation response and the potential occurrence of atherosclerosis and CAD. In this study, we aimed to examine the levels of COX-2 expression of peripheral blood monocyte and the inflammatory indexes in CAD patients, We further aimed to assess the association of COX-2 with MPA formation, and the predicting significance of COX-2 for CAD.

\section{Methods}

\section{Participants}

A total of 66 patients with CAD were recruited in this prospective study, the patients included 41 males and 25 females aged from 34 to 78 years old, and were hospitalized in Zhejiang Provincial People's Hospital between January 2018 and July 2018. There were 37 patients with stable angina (SA), including 24 males and 13 females aged from 34 to 72 years old, and 29 patients with unstable angina (UA), including 19 males and 10 females aged from 37 to 78 years old. The inclusion criteria for patients were as follows: (I) coronary atherosclerotic heart disease; (II) first diagnosed inpatients; (III) before drug treatment. The exclusion criteria were as follows: (I) acute myocardial infarction; (II) infection; (III) primary hypertension; (IV) primary liver or kidney dysfunction; (V) malignancies; (VI) cerebrovacular diseases; (VII) diabetes mellitus; (VIII) history of thrombosis; (IX) pregnant woman; (X) taking antithrombotic drugs and statins in one month before sample collecting; (XI) taking any other drug in one week before sample collecting. All of the patients were the Hans Chinese and diagnosed according to the ACCF guidelines for the diagnosis and management of patients with stable ischemic heart disease and UA $(10,11)$. At the same time, 30 healthy subjects who were matched for age, sex and race, were included in the analysis.

\section{Sample collection}

Peripheral blood samples were collected in trisodium citrate-, dipotassium EDTA- or lithium heparin-containing tubes and anticoagulant-free tubes, respectively, after an overnight fast on the morning before treatment. The samples were further used for the isolation of monocytes, the measurements of MPA formation, the analysis of white blood cells (WBCs) and high-sensitive $\mathrm{C}$ reactive protein (hs-CRP), the detection of COX-2 expression, and the assay of MCP-1, respectively.

\section{Isolation and treatment of peripheral blood monocytes}

Fresh peripheral blood was taken into vacuum tubes containing lithium heparin (19 USP U/mL) (BD company, Franklin Lakes, NJ, USA). The peripheral blood mononuclear cells (PBMCs) were isolated using FicollHypaque 1.077 g/mL (Dingguo Changsheng Co., Ltd., Beijing, China), as previously described (12). Monocyte was isolated from PBMC by cell culture flask adherence as plastic adherence method. For monocyte isolation by adherence, $10-15 \times 10^{6}$ PBMC per flask were seeded into RPMI 1640 medium (Life Technologies, Carlsbad, CA, USA; 23400021) containing 10\% FBS (Life Technologies, Carlsbad, CA, USA; 10099) and incubated at $37{ }^{\circ} \mathrm{C}$ for 2 hours in a humidified atmosphere containing $5 \% \mathrm{CO}_{2}$ (Thermo, Waltham, MA, USA; 3111). Non-adherent cells were removed and the adherent cells were carefully washed twice with the medium. And the removed non-adherent cells were re-treated according to the abovementioned process to obtain more adherent cells. Subsequently, monocyte suspension was collected, and the CD14 positive cells were measured by a flow cytometer (FC500-MCL, 
Beckman-Coulter Company, Brea, CA, USA) to confirm the concentrations of monocytes, and a CD14 expression rate of more than $90 \%$ indicated the appropriate concentrations of monocytes. Finally, the monocyte counts were adjusted to $5 \times 10^{5} / \mathrm{mL}$, and the suspension was prepared for the following detection.

\section{Measurement of MPA formation}

For the measurement of MPA, the whole blood with citrate was incubated with CD14-PE rabbit anti-human monoclonal antibodies and CD41-FITC mouse antihuman monoclonal antibodies (Immunotech, Glendale, CA, USA) and the isotype matched controls for 20 mins in a dark cabinet at room temperature. After performing the detection with a flow cytometry (FCM, FC500-MCL, Beckman-Coulter Company, Brea, CA, USA), the MPA formation was gated by identifying both CD14 and CD41 positive cells.

\section{Assays of inflammatory indexes}

Mixed whole blood with EDTA- $\mathrm{K}_{2}$ was first analyzed for the counts of WBCs, neutrophils and lymphocytes by an automatic hematological analyzer and commercially available reagents (BC-6900, Mindray Inc., Shenzhen, China). Subsequently, the neutrophil-to-lymphocyte ratio (NLR) was calculated. At the same time, hs-CRP concentrations of whole blood were measured by a specific protein analyzer (CRP-M100, Mindray Inc., Shenzhen, China). For the assay of MCP-1, the anticoagulant-free blood was centrifuged at $1,500 \times \mathrm{g}$ for $10 \mathrm{~min}$, and the supernatant was collected to detect MCP-1 level by an ELISA kit (Bioscience) according to the manufacturer's instructions. Briefly, $100 \mu \mathrm{L}$ of samples and standards were added into the enzyme-linked immunosorbent assay (ELISA) plate and incubated at $37^{\circ} \mathrm{C}$ for $90 \mathrm{~min}$. After washing with cleaning solution for 5 times, biotinylated antiMCP-1 antibody was added into the wells and incubated for another $60 \mathrm{~min}$ at $37^{\circ} \mathrm{C}$. Subsquently, enzyme conjugates were added into the mixture and standed for $30 \mathrm{~min}$ at $37{ }^{\circ} \mathrm{C}$ after washing for 5 times. Finally, $100 \mu \mathrm{L}$ of chromogenic substrate TMB was added and incubated for 15 mins at $37{ }^{\circ} \mathrm{C}$ in darkness. After terminating the reaction, the optical density (OD) values of samples were detected at the wavelength of $450 \mathrm{~nm}$ by a microplate reader (Sigma, St. Louis, MO, USA; 1-15K). Eventually, MCP1 concentrations were calculated based on the equation of standard curve.

\section{Detection of COX-2 mRNA expression}

Total RNA from the samples of monocytes suspension were isolated in the light of the protocol of Trizol reagent (Roche, Basel, Switzerland). One microgram $(1 \mu \mathrm{g})$ total RNA was reversely transcribed into cDNA using SuperRT cDNA Synthesis Kit cDNA (CwBiotech, CW0741S, China). The expression level of COX2 gene was evaluated by performing real-time PCR utilizing UltraSYBR Mixture (High ROX) (CwBiotech, CW2602M, China). Primer sequences of COX-2 are as follows: forward, AACGATCCCTCCCTTACCAT; reverse, GTTTAGACGTCCGGGAATTG. The $\beta$-actin (forward, GATGAGATTGGCATGGCTTT; reverse, GTCACCTTCACCGTTCCAGT) was served as the internal control. Relative expression of COX-2 gene was calculated according to $2^{-\Delta \Delta C t}$ method.

\section{Analysis of COX-2 protein expression}

All of the samples of monocytes suspension were centrifuged and the pellet was resuspended using RIPA cell lysis solution containing protease inhibitors (Beijing Dingguo Changsheng Inc., China). After shake vigorously for 30 mins at $4{ }^{\circ} \mathrm{C}$, the lysis solution was centrifuged at $12,000 \times \mathrm{g}$ for $10 \mathrm{mins}$ at $4{ }^{\circ} \mathrm{C}$. The supernatant was then collected, and the quantitative protein analysis was performed by a BCA kit according to the manufacturer's instructions. Total $30 \mu \mathrm{g}$ of protein was used to perform SDS-PAGE assay. COX-2 antibody (rabbit anti-human monoclonal antibody) was obtained from Abcam, and relative protein analysis was processed based on Quantity One software.

\section{Statistical analysis}

Data were first performed test of normality by KolmogorovSmirnov test (K-S test), and normal distributed data were presented as the mean \pm standard deviation (SD). Relative protein level was measured using Quantity One software. The difference of normal distributed results was compared with Student's $t$-test. The correlation was analyzed by Pearson correlation test, and Logistic regression analysis was used for risk assessment on CAD. All statistical analyses were performed with SPSS software (version 17.0). P value of less than 0.05 was considered as statistically significant. 
Table 1 Comparison of inflammatory indicators levels between CAD patients and controls

\begin{tabular}{lccccc}
\hline Subjects & Number & hs-CRP $(\mathrm{mg} / \mathrm{L})$ & MCP-1 $(\mathrm{ng} / \mathrm{L})$ & WBC $\left(\times 10^{9} / \mathrm{L}\right)$ & NLR \\
\hline Controls & 30 & $0.91 \pm 0.89$ & $101.2 \pm 21.2$ & $5.81 \pm 2.30$ & $2.09 \pm 1.22$ \\
CAD patients & 66 & $6.32 \pm 5.61^{\star}$ & $181.3 \pm 34.7^{\star}$ & $9.33 \pm 3.52^{\star}$ & $3.21 \pm 2.28^{\star}$ \\
SA patients & 37 & $3.33 \pm 2.15$ & $145.3 \pm 22.1$ & $7.89 \pm 2.78$ & $2.76 \pm 2.01$ \\
UA patients & 29 & $10.13 \pm 9.32^{\Delta}$ & $227.2 \pm 27.4^{\Delta}$ & $11.17 \pm 3.20^{\Delta}$ & $3.78 \pm 1.69^{\Delta}$ \\
\hline
\end{tabular}

Data were presented as the mean $\pm \mathrm{SD}$. * indicates CAD patients vs. controls; ${ }^{\star}$, indicates UA patients vs. SA patients. CAD, coronary artery disease; SA, stable angina; UA, unstable angina; hs-CRP, high-sensitive C reactive protein; WBC, white blood cell; MCP-1, monocyte chemoattractant protein-1; NLR, neutrophil-to-lymphocyte ratio; SD, standard deviation.

\section{Results}

\section{Levels of inflammatory indexes in patients and controls}

Levels of total four inflammatory indexes were observed in $66 \mathrm{CAD}$ patients and 30 controls. Concentrations of hs-CRP and MCP-1, the WBC counts and the NLR were significantly higher in patients with CAD compared with controls $(\mathrm{P}<0.001)$. Furthermore, compared with SA patients, UA patients demonstrated increased concentrations of hs-CRP and MCP-1, WBC counts and the NLR $(\mathrm{P}<0.001)$ (Table 1).

\section{Levels of MPA formation and COX-2 expression in CAD patients}

To compare and assess the difference of MPA formation between patients with SA and UA, a FCM was used to determine the rates of MPA formation. We found that both SA and UA patients had higher MPA rates than those of the controls $(22.1 \% \pm 3.1 \%$ and $30.2 \% \pm 4.1 \%$ vs. $10.2 \% \pm 1.3 \%$; $\mathrm{P}<0.001$, respectively), and UA patients demonstrated higher MPA rates than SA patients $(\mathrm{P}<0.01)$ (Figure $1 A)$. Furthermore, all of the patients with SA and UA showed enhanced expression levels of COX-2 mRNA and protein compared with the controls $(\mathrm{P}<0.001$, respectively), and UA patients also demonstrated enhanced COX-2 expression compared with SA patients $(\mathrm{P}<0.01$; respectively) (Figure 1B,C).

\section{Association of $C O X-2$ expression with MPA formation rate}

All of the CAD patients included 18 individuals with enhanced COX-2 expression and 48 ones without, and the patients with enhanced COX-2 expression also demonstrated higher MPA formation rates than those without $(28.5 \% \pm 6.6 \%$ vs. $18.1 \% \pm 5.3 \% ; \mathrm{P}<0.01)$
(Figure 2A). Moreover, all of the patients were constituted of 45 individuals with increased MPA formation rates and 21 ones without, which the former had enhanced COX-2 expression compared with the latter $(0.53 \pm 0.04$ vs. $0.32 \pm 0.03, \mathrm{P}<0.001$ ) (Figure $2 B$ ). The correlation analysis further indicated that the levels of $\mathrm{COX}-2$ protein expression was significantly related to the rates of MPA formation $\left(\mathrm{R}^{2}=0.4933, \mathrm{P}<0.01\right)$ (Figure $2 C$ ).

\section{Hazard Regression analysis for CAD}

Univariate regression analysis revealed that age, COX-2 expression, MPA formation, and the inflammation indexes were the risk factors for CAD. However, multivariate analysis demonstrated that COX-2 expression, MPA formation, hs-CRP, and MCP-1 were the independent risk factors for CAD. At the same time, COX-2 had a higher odds ratio (OR) value [6.322, 95\% confidence interval (CI): 4.544-8.978] than all of the other indexes (Table 2).

\section{Discussion}

The activation-dependent conformational change in monocyte surface Mac-1 contributes to the atherogenesis and the development of other inflammatory diseases (13). As we know, a systemic inflammatory response would demonstrate increased $\mathrm{WBC}$ counts and the release of proinflammatory cytokines/molecule; thus, these indexes can be used as the inflammatory indicators. Monocyte activation involves in a complicated process, and can display the enhancement of chemotactic and adhesive function, which is characterized by the release of inflammatory factors such as MCP-1 (14). The present study showed that the concentrations of MCP-1 and hs-CRP, WBC counts and the NLR were significantly increased in patients with CAD, which indicated that the change trend of inflammatory 
A

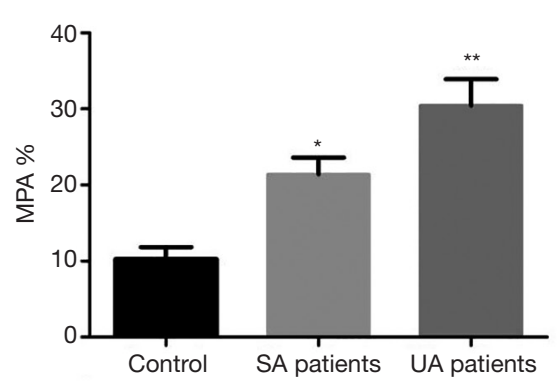

B

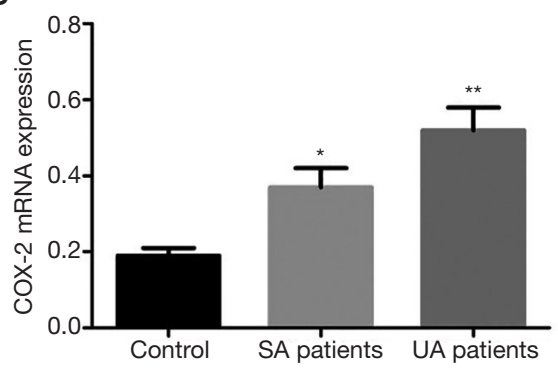

C

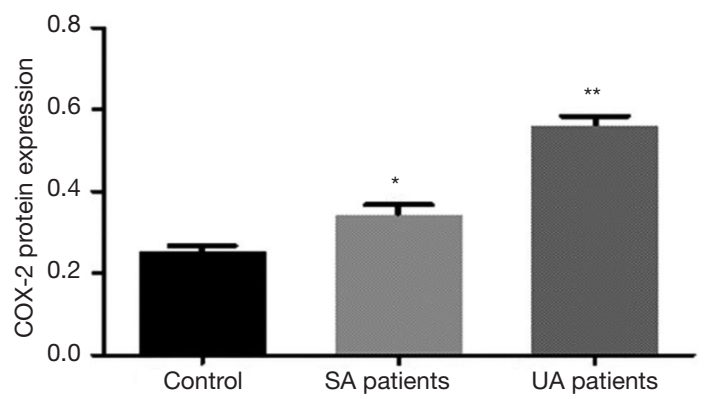

COX-2

$\beta$-actin

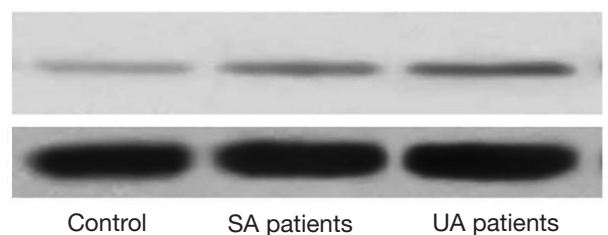

Figure 1 Levels of MPA formation and COX-2 expression in UA and SA patients. Data were presented as the mean \pm SD. * or ** indicates SA or UA patients $v s$. controls, respectively; *, $\mathrm{P}<0.01$; **, $\mathrm{P}<0.001$. SA, stable angina; UA, unstable angina; MPA, monocyte platelet aggregate; COX-2, cyclooxygenase-2; SD, standard deviation.

A

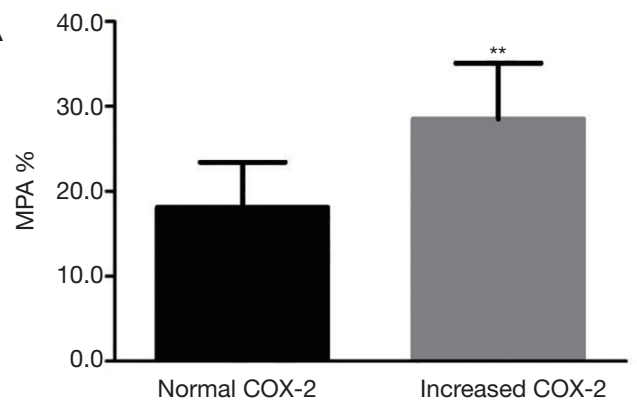

C

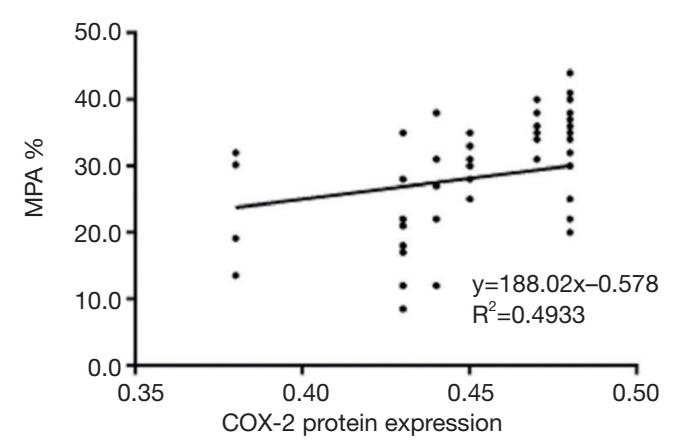

B

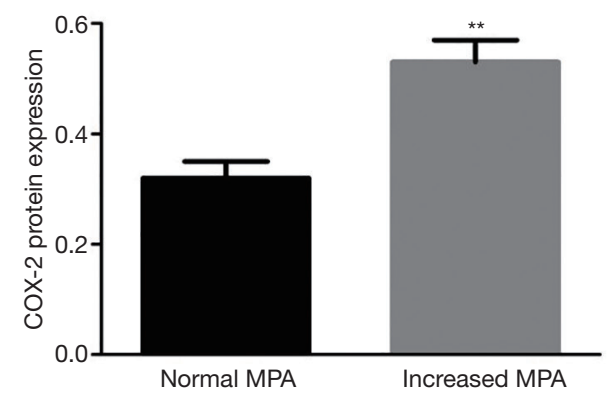

Figure 2 Association of COX-2 protein expression with MPA formation in CAD patients. (A) MPA formation rate in different expression level of COX-2 protein; (B) expression level of COX-2 protein in different MPA formation rate; (C) correlation of COX-2 expression levels with MPA formation rate. ${ }^{* *}$ indicates increased COX-2 or increased MPA groups vs. normal COX-2 or normal MPA groups, respectively; **, $\mathrm{P}<0.001$. CAD, coronary artery disease; MPA, monocyte platelet aggregate; COX-2, cyclooxygenase-2. 
Table 2 Results of Logistic regression analysis for CAD

\begin{tabular}{|c|c|c|c|c|}
\hline Variables & \multicolumn{2}{|c|}{ Univariate analysis } & \multicolumn{2}{|c|}{ Multivariate analysis } \\
\hline Gender & $0.845(0.566-1.032)$ & $>0.05$ & $0.769(0.501-0.01)$ & $>0.05$ \\
\hline Age & $1.201(1.045-1.602)$ & $<0.01$ & $1.101(0.931-1.200)$ & $>0.05$ \\
\hline cox-2 & $7.812(5.432-10.321)$ & $<0.001$ & $6.322(4.544-8.978)$ & $<0.001$ \\
\hline hs-CRP & $5.321(3.221-8.012)$ & $<0.001$ & $4.221(2.345-5.865)$ & $<0.001$ \\
\hline MCP-1 & $6.442(4.320-8.777)$ & $<0.001$ & $5.235(3.301-7.001)$ & $<0.001$ \\
\hline WBC & 2.334 (1.476-3.702) & $<0.001$ & $1.255(0.802-3.033)$ & $>0.05$ \\
\hline NLR & $4.561(3.022-6.890)$ & $<0.001$ & $4.011(2.799-6.121)$ & $<0.001$ \\
\hline
\end{tabular}

CAD, coronary artery disease; MPA, monocyte-platelet aggregate; COX-2, cyclooxygenase-2; hs-CRP, high-sensitive C reactive protein; MCP-1, monocyte chemoattractant protein-1; WBC, white blood cell; NLR, neutrophil-to-lymphocyte ratio; OR, odds ratio; Cl, confidence interval.

indexes levels was consistent with one another, and may also be accordance with the basic manifestation of the inflammatory response in the patients. Additionally, patients with UA had higher levels of inflammatory indexes than those with SA, which further suggests that the inflammatory response induced by different factors may be related to the progression of atherosclerosis and the severity of CAD. Therefore, this finding suggests that the inflammatory response was closely related to the occurrence of CAD, and may further increase the risk for the occurrence and development of CAD, and the measurements of inflammatory indexes will help to predict the risk of CAD and further assess the disease condition.

MPA formation alters the phenotype of monocytes along with the increase of Mac-1 expression and activation, and the enhanced Mac-1 can bind to platelet fibrinogen receptor (glycoprotein IIb/IIIa receptor) and maintain the adhesion of platelets to monocytes and monocytes activation in turn (15). Linden et al. (16) reported that MPA is significantly elevated in acute and unstable atherothrombosis, implying that measurements of MPA might be used as an evaluating indicator in early stage of atherosclerotic disease. Thus, this indicates that the MPA formation and the subsequent monocytes activation play an extremely important role in the occurrence and development of atherosclerotic diseases, and may be closely related to the inflammatory response and cardiovascular disease. The present study demonstrated that both SA and UA patients had increased rates of MPA formation, and UA patients exhibited higher rates than SA patients, which suggests that MPA may be associated with the development of CAD, and elevated MPA rates probably indicates a higher risk for the progression of CAD. Some studies indicate that unregulated COX-2 expression can influence chronic inflammatory conditions, including atherosclerosis and its complications $(17,18)$. Our previous studies also revealed that papain has some inhibitory effects on platelet activation (19), MPA formation and the expression of COX-2 as well as the secretion of MCP-1 in THP-1 and peripheral blood monocytes $(9,20)$. Therefore, COX2 may be the target of inflammation regulation, and the enhanced COX-2 expression may be the key that activated monocytes induced by MPA formation facilitate the inflammatory response, potentially leading to the development of atherosclerosis. Thus, if COX-2 expression can be inhibited effectively, it will be helpful to prevent and treat atherosclerotic diseases. The present study revealed that both SA and UA patients demonstrated enhanced COX-2 expression in monocyte, and UA patients had higher expression than SA patients, which was consistent with the increase of MPA formation. This study further showed that the levels of COX-2 expression in monocytes was positively correlated with the rates of MPA formation and the levels of other inflammatory indexes in patients with CAD. Thus, our results revealed that enhanced COX2 expression in monocytes was closely correlated with the degree of inflammatory response induced by MPA formation, and further suggest that COX-2 is the key substance in the development and progression of CAD. Therefore, this finding suggests that, $\mathrm{COX}-2$, as a regulator, 
plays an important role in the inflammatory regulation of monocyte activation, which may be helpful to predict the progression of coronary heart disease.

Several different pro-inflammatory cytokines in initially healthy people are associated with the risk for CAD outcomes (21), and inflammatory markers may play important role in prediction and risk assessment on coronary heart disease (22). Previous studies have indicated that WBC counts and the subtypes have been implicated as the predictors of CAD (23), and its mortality (24), and CRP is related to the severity of CAD and may be an independent risk factor for acute coronary syndrome (25). Therefore, inflammatory indexes would play some important roles in the assessment on the risk and prognosis of CAD. In the present study, hazard regression analysis showed that the inflammatory regulator COX-2 was an independent risk factor with a high OR value of 6.332 for $\mathrm{CAD}$, which suggests that the enhanced COX-2 expression in monocytes can significantly increase the risk for CAD. Therefore, this finding further suggests that $\mathrm{COX}-2$ can be used as the predictor of CAD.

To the best of our knowledge, there may be at least two limitations of the present study. Firstly, in our study, there probably were some patients with chronic inflammation who were not be diagnosed before samples collection. Therefore, the data from those patients might cause some inexact results and potentially influence the predicting power of COX-2. Secondly, COX-2 detection has no uniform standard and was widely used. However, in this study, we have measured COX-2 protein and mRNA expression by western-blotting and reverse transcriptionpolymerase chain reaction (RT-PCR) assays, respectively, and our results also displayed significantly clinical significance of COX-2 measurements. Therefore, although there were some limitations, the present study also revealed the predicting significance of monocyte COX-2 expression in CAD patients.

In conclusion, the present study evaluated the COX-2 expression level of peripheral blood monocytes in patients with $\mathrm{CAD}$, and revealed that the enhancement of COX-2 expression is associated with the formation of MPA, and suggests that COX-2 can be used as an independent risk predictor for $\mathrm{CAD}$.

\section{Acknowledgments}

We thank all those who supported us in volunteer recruitment and help us to manage experimental data.
Funding: This work was supported by the Natural Science Foundation of Zhejiang Province (Grant No. LY17H080007).

\section{Footnotes}

Conflicts of Interest: The authors have no conflicts of interest to declare.

Ethical Statement: The authors are accountable for all aspects of the work in ensuring that questions related to the accuracy or integrity of any part of the work are appropriately investigated and resolved. This study was approved by the Ethics Committee of Zhejiang Provincial People's Hospital (ID of ethics approval: 2017KY111). The study outcomes will not affect the future management of the patients. The use of human blood samples was in accordance with the legislation in China. Informed consent was obtained from the controls and patients or their guardian, and the study was approved by the institutional review board of the hospital.

\section{References}

1. Shoenfeld Y, Sherer Y, Harats D. Artherosclerosis as an infectious, inflammatory and autoimmune disease. Trends Immunol 2001;22:293-5.

2. Li X, Fang P, Li Y, et al. Mitochondrial reactive oxygen species mediate lysophosphatidylcholine-induced endothelial cell activation. Arterioscler Thromb Vasc Biol 2016;36:1090-100.

3. Haynes A, Linden MD, Robey E, et al. Relationship between monocyte-platelet aggregation and endothelial function in middle-aged and elderly adults. Physiol Rep 2017;5:e13189.

4. Dixon DA, Tolley ND, Bemis-Standoli K, et al. Expression of COX-2 in platelet-monocyte interactions occurs via combinatorial regulation involving adhesion and cytokine signaling. J Clin Invest 2006;116:2727-38.

5. Japp AG, Chelliah R, Tattersall L, et al. Effect of PSI697, a novel P-selectin inhibitor, on platelet-monocyte aggregate formation in humans. J Am Heart Assoc 2013;2:e06007.

6. Neumann FJ, Marx N, Gawaz M, et al. Induction of cytokine expression in leukocytes by binding of thrombinstimulated platelets. Circulation 1997;95:2387-94.

7. Passacquale G, Vamadevan P, Pereira L, et al. Monocyteplatelet interaction induces a pro-inflammatory phenotype 
in circulating monocytes. PLoS One 2011;6:e25595.

8. Pettus BJ, Bielawski J, Porcelli AM, et al. The sphingosine kinase 1/sphingosine-1-phosphate pathway mediates COX-2 induction and PGE2 production in response to TNF-alpha. FASEB J 2003;17:1411-21.

9. Fei X, Yuan W, Zhao Y, et al. Papain ameliorates the MPAs formation-mediated activation of monocytes by inhibiting Cox-2 expression via regulating the MAPKs and PI3K/Akt signal pathway. Biomed Res Int 2018;2018:3632084.

10. Fihn SD, Gardin JM, Abrams J, et al. 2012 ACCF/ AHA/ACP/AATS/PCNA/SCAI/STS guideline for the diagnosis and management of patients with stable ischemic heart disease: a report of the American College of Cardiology Foundation/American Heart Association task force on practice guidelines, and the American College of Physicians, American Association for Thoracic Surgery, Preventive Cardiovascular Nurses Association, Society for Cardiovascular Angiography and Interventions, and Society of Thoracic Surgeons. Circulation 2012;126:e354-471.

11. Wright RS, Anderson JL, Adams CD, et al. 2011 ACCF/ AHA focused update incorporated into the ACC/AHA 2007 guidelines for the management of patients with unstable angina/non-ST-elevation myocardial infarction: a report of the American College of Cardiology Foundation/ American Heart Association task force on practice guidelines developed in collaboration with the American Academy of Family Physicians, Society for Cardiovascular Angiography and Interventions, and the Society of Thoracic Surgeons. J Am Coll Cardiol 2011;57:e215-367.

12. Böyum A. Separation of leukocytes from blood and bone marrow. Introduction. Scand J Clin Lab Invest Suppl 1968;97:7.

13. Hui H, Fuller K, Erber WN, et al. Measurement of monocyte-platelet aggregates by imaging flow cytometry. Cytometry A 2015;87:273-8.

14. van Gils JM, Zwaginga JJ, Hordijk PL. Molecular and functional interactions among monocytes, platelets, and endothelial cells and their relevance for cardiovascular

Cite this article as: Huang Q, Fei X, Li S, Xu C, Tu C, Jiang L, Wo M. Predicting significance of COX-2 expression of peripheral blood monocyte in patients with coronary artery disease. Ann Transl Med 2019;7(18):483. doi: 10.21037/ atm.2019.08.75 diseases. J Leukoc Biol 2009;85:195-204.

15. Barnard MR, Linden MD, Frelinger AL 3rd, et al. Effects of platelet binding on whole blood flow cytometry assays of monocyte and neutrophil procoagulant activity. J Thromb Haemost 2005;3:2563-70.

16. Linden MD, Furman MI, Frelinger AL 3rd, et al. Indices of platelet activation and the stability of coronary artery disease. J Thromb Haemost 2007;5:761-5.

17. Cipollone F, Rocca B, Patrono C. Cyclooxygenase-2 expression and inhibition in atherothrombosis. Arterioscler Thromb Vasc Biol 2004;24:246-55.

18. Koki A, Khan NK, Woerner BM, et al. Cyclooxygenase-2 in human pathological disease. Adv Exp Med Biol 2002;507:177-84.

19. Fei XM, Zhou YJ, Qi JW, et al. In vitro inhibitory effects of papain on platelet aggregation. Chin J Pharmacol Ther 2009;14:906-11.

20. Jiang L, Fei XM, Cheng ML, et al. Effects of papain on monocyte-platelet aggregates formation and monocyte activation induced by monocyte-platelet aggregates. Chin J Pharmaco Ther 2015;20:289-92.

21. Kaptoge S, Seshasai SR, Gao P, et al. Inflammatory cytokines and risk of coronary heart disease: new prospective study and updated meta-analysis. Eur Heart J 2014;35:578-89.

22. Li H, Sun K, Zhao R, et al. Inflammatory biomarkers of coronary heart disease. Front Biosci (Landmark Ed) 2017;22:504-15.

23. Madjid M, Fatemi O. Components of the complete blood count as risk predictors for coronary heart disease: indepth review and update. Tex Heart Inst J 2013;40:17-29.

24. Shah N, Parikh V, Patel N, et al. Neutrophil lymphocyte ratio significantly improves the Framingham risk score in prediction of coronary heart disease mortality: insights from the National Health and Nutrition Examination Survey-III. Int J Cardiol 2014;171:390-7.

25. Badimon L, Peña E, Arderiu G, et al. C-reactive protein in atherothrombosis and angiogenesis. Front Immunol 2018;9:430. 